\title{
Nanovolcano microelectrode arrays: toward long- term on-demand registration of transmembrane action potentials by controlled electroporation
}

\author{
Benoît X. E. Desbiolles (1)' Etienne de Coulon², Nicolas Maïno', Arnaud Bertsch (1)', Stephan Rohr ${ }^{2}$ and \\ Philippe Renaud (1D)
}

\begin{abstract}
Volcano-shaped microelectrodes (nanovolcanoes) functionalized with nanopatterned self-assembled monolayers have recently been demonstrated to report cardiomyocyte action potentials after gaining spontaneous intracellular access. These nanovolcanoes exhibit recording characteristics similar to those of state-of-the-art micro-nanoelectrode arrays that use electroporation as an insertion mechanism. In this study, we investigated whether the use of electroporation improves the performance of nanovolcano arrays in terms of action potential amplitudes, recording durations, and yield. Experiments with neonatal rat cardiomyocyte monolayers grown on nanovolcano arrays demonstrated that electroporation pulses with characteristics derived from analytical models increased the efficiency of nanovolcano recordings, as they enabled multiple on-demand registration of intracellular action potentials with amplitudes as high as $62 \mathrm{mV}$ and parallel recordings in up to $~ 76 \%$ of the available channels. The performance of nanovolcanoes showed no dependence on the presence of functionalized nanopatterns, indicating that the tip geometry itself is instrumental for establishing a tight seal at the cell-electrode interface, which ultimately determines the quality of recordings. Importantly, the use of electroporation permitted the recording of attenuated cardiomyocyte action potentials during consecutive days at identical sites, indicating that nanovolcano recordings are nondestructive and permit long-term on-demand recordings from excitable cardiac tissues. Apart from demonstrating that less complex manufacturing processes can be used for next-generation nanovolcano arrays, the finding that the devices are suitable for performing on-demand recordings of electrical activity from multiple sites of excitable cardiac tissues over extended periods of time opens the possibility of using the devices not only in basic research but also in the context of comprehensive drug testing.
\end{abstract}

\section{Introduction}

Cell membrane electroporation is a well-established method for gaining access to the cell interior. This technique is based on the application of voltage pulses of sufficient magnitude that cause dielectric breakdown of the cell membrane, thereby inducing the formation of

\footnotetext{
Correspondence: Benoît X. E. Desbiolles (benoit.desbiolles@epfl.ch)

'Laboratory of Microsystems LMIS4, Ecole Polytechnique Fédérale de

Lausanne, Lausanne, Switzerland

${ }^{2}$ Department of Physiology, Laboratory of Cellular Optics II, University of Bern, Bern, Switzerland

These authors contributed equally: Benoît X. E. Desbiolles, Etienne de Coulon

These authors jointly supervised this work: Stephan Rohr, Philippe Renaud
}

nanopores $^{1,2}$. Over the past decades, biological and medical applications of electroporation ${ }^{3,4}$ have mainly focused on therapeutic gene transfer ${ }^{5-7}$, extraction of molecules $^{8-10}$, and electrochemotherapy ${ }^{11-13}$. More recently, electroporation has become a method of choice for imparting micro-nanoelectrodes with intracellular access capabilities. In the context of nanoelectrophysiology, electroporation combined with multielectrode arrays (MEAs) has the potential to overcome some limitations of the current gold standard, i.e., whole-cell patch-clamp recording. This technology offers only limited throughput, the recording duration is generally short, and performing

\section{(c) The Author(s) 2020}

(c) (i) Open Access This article is licensed under a Creative Commons Attribution 4.0 International License, which permits use, sharing, adaptation, distribution and reproduction cc) in any medium or format, as long as you give appropriate credit to the original author(s) and the source, provide a link to the Creative Commons license, and indicate if changes were made. The images or other third party material in this article are included in the article's Creative Commons license, unless indicated otherwise in a credit line to the material. If material is not included in the article's Creative Commons license and your intended use is not permitted by statutory regulation or exceeds the permitted use, you will need to obtain permission directly from the copyright holder. To view a copy of this license, visit http://creativecommons.org/licenses/by/4.0/. 
experiments is associated with high personnel costs ${ }^{14}$. By contrast, the use of electroporation in conjunction with arrays of nanoneedle-like electrodes recently enabled simultaneous recordings of intracellular action potentials (APs) from hundreds of electrotonically coupled rat cardiomyocytes over several days ${ }^{15-17}$ as well as from thousands of neurons ${ }^{18}$. Recordings made with electrodes of various geometries such as micromushrooms ${ }^{19-22}$ or nanotubes $^{23}$ demonstrated similar electrophysiological characteristics. Current limitations of these technologies include substantial signal attenuation, short intracellular recording durations, and low yield.

In our recent work, we described a novel type of nanopatterned volcano-shaped hollow microelectrode called a "nanovolcano," which is suited to perform attenuated transmembrane voltage measurements from networks of cardiomyocytes without the need for active electroporation ${ }^{24}$. The inner surface of the slightly conical hollow nanovolcanoes having a diameter of $2 \mu \mathrm{m}$ consists of a large three-dimensional Pt electrode that offers lowimpedance access to the cell interior. The outer surface of the nanovolcano is insulated by a 50 -nm-thick $\mathrm{SiO}_{2}$ layer. A 10-20-nm-thick gold layer flanked by two $\mathrm{Ti}$ layers is intercalated between the $\mathrm{Pt}$ and $\mathrm{SiO}_{2}$ layers that form the nanovolcano wall. The gold layer is exposed along the upper rim of the nanovolcano and is functionalized with alkanethiol self-assembled monolayers ${ }^{25}$. This functionalization has been previously shown in planar electrodes to support the formation of gigaohm seals lasting several days ${ }^{26}$. Further facilitating seal formation, as previously reported for nanopillars ${ }^{27,28}$, the 100-nm-thin upper rim of the nanovolcano induces high-curvature regions in the membrane of contacting cells, which are expected to maximize the coupling between the cell and the electrode.

We have shown that nanovolcanoes permit the recording of attenuated transmembrane APs from spontaneously active monolayer cultures of primary neonatal rat cardiomyocyte cultures without applying active electroporation $^{24}$. The yield of AP recordings with amplitudes of $6.6 \pm 1.8 \mathrm{mV}(N=3087)$ was $15 \pm 11 \%(N=4)$, and continuous recordings for $18.2 \pm 21 \mathrm{~min}(N=16)$ were achieved. Static charge-induced electroporation was suspected to be the main mechanism responsible for the spontaneously occurring sporadic intracellular access of nanovolcanoes. If this hypothesis is correct, controlled electroporation applied to all nanovolcanoes of the array should improve the yield of electrodes showing intracellular access. Moreover, controlled electroporation may improve the amplitudes of the recorded signals by reducing the access resistance and may permit longer duration measurements. Hypothetically, the quality of recordings may furthermore benefit from the thiol functionalization of the gold ring that is in contact with the cells.
In the present study, we investigated these questions using spontaneously active monolayer cultures of neonatal rat ventricular cardiomyocytes ${ }^{29}$. Optimal electroporation waveforms and frequencies were derived from a theoretical model of the cell-electrode interface. The usefulness of thiol functionalization was tested by comparing the performance of nanovolcanoes produced with and without this feature. Controlled electroporation with nanovolcano arrays enabled on-demand intermittent intracellular access for 2 days, with recorded AP amplitudes (APAs) reaching $62 \mathrm{mV}$ and yields as high as $\sim 76 \%$. Nonpatterned nanovolcanoes showed similar performance compared with that of nanovolcanoes containing functionalized gold nanorings.

\section{Results}

\section{Electroporation modeling}

A theoretical model representing the cell-electrode interface was established to analytically determine the optimal voltage waveform and frequency necessary to reliably electroporate cell membranes with nanovolcano electrodes. Figure 1a illustrates the electrical equivalent circuit of the cell-nanovolcano interface. The electrode-electrolyte interface is composed of a nonlinear resistance, $R_{\mathrm{CT}}$, that represents faradaic charge-transfer secondary to redox reactions, in parallel with a constant phase element, $C P E_{\mathrm{DL}}$, that represents the double layer formed by the accumulation of opposite charges at the electrode-electrolyte interface underlying capacitive charge-transfer. The stray capacitance, $C_{\text {Stray }}$, denotes the capacitive current leaks along the insulated conductive tracks. A complete electrochemical characterization of the electrode-electrolyte interface was described in detail in our previous work ${ }^{24}$. The junctional cell membrane covering the microelectrode is modeled by its junctional capacitance, $C_{j}$, and the parallel resistance, $R_{j}$. The nonjunctional membrane impedance is neglected. The seal resistance, $R_{\text {Seal }}$, depicts the current leaks at the cell-electrode interface. To simplify the model and consider the worst-case scenario, the resting membrane potential of the cell was not taken into account. Accordingly, $V_{j}$ directly reflects the potential applied across the cell membrane.

To induce dielectric breakdown of cell membranes, the junctional potential across the cell membrane $\left(V_{j}\right)$ must reach $300-400 \mathrm{mV}^{30,31}$. Due to the voltage divider formed by the electrode impedance at the electrode-electrolyte interface $\left(R_{\mathrm{CT}} \| \mathrm{CPE}_{\mathrm{DL}}\right)$ and the impedance representing the remainder of the cell-electrode interface $\left(R_{\text {Seal }}\left\|C_{j}\right\|\right.$ $\left.R_{j}\right), V_{j}$ is attenuated compared with the electroporation voltage $\left(V_{\mathrm{EP}}\right)$ applied at the terminals of the device. This attenuation should be minimized to keep $V_{\mathrm{EP}}$ low and prevent local hydrolysis in the junctional space. Figure $1 \mathrm{~b}$ depicts the evolution of the attenuation ratio $V_{j} / V_{\mathrm{EP}}$ as a 

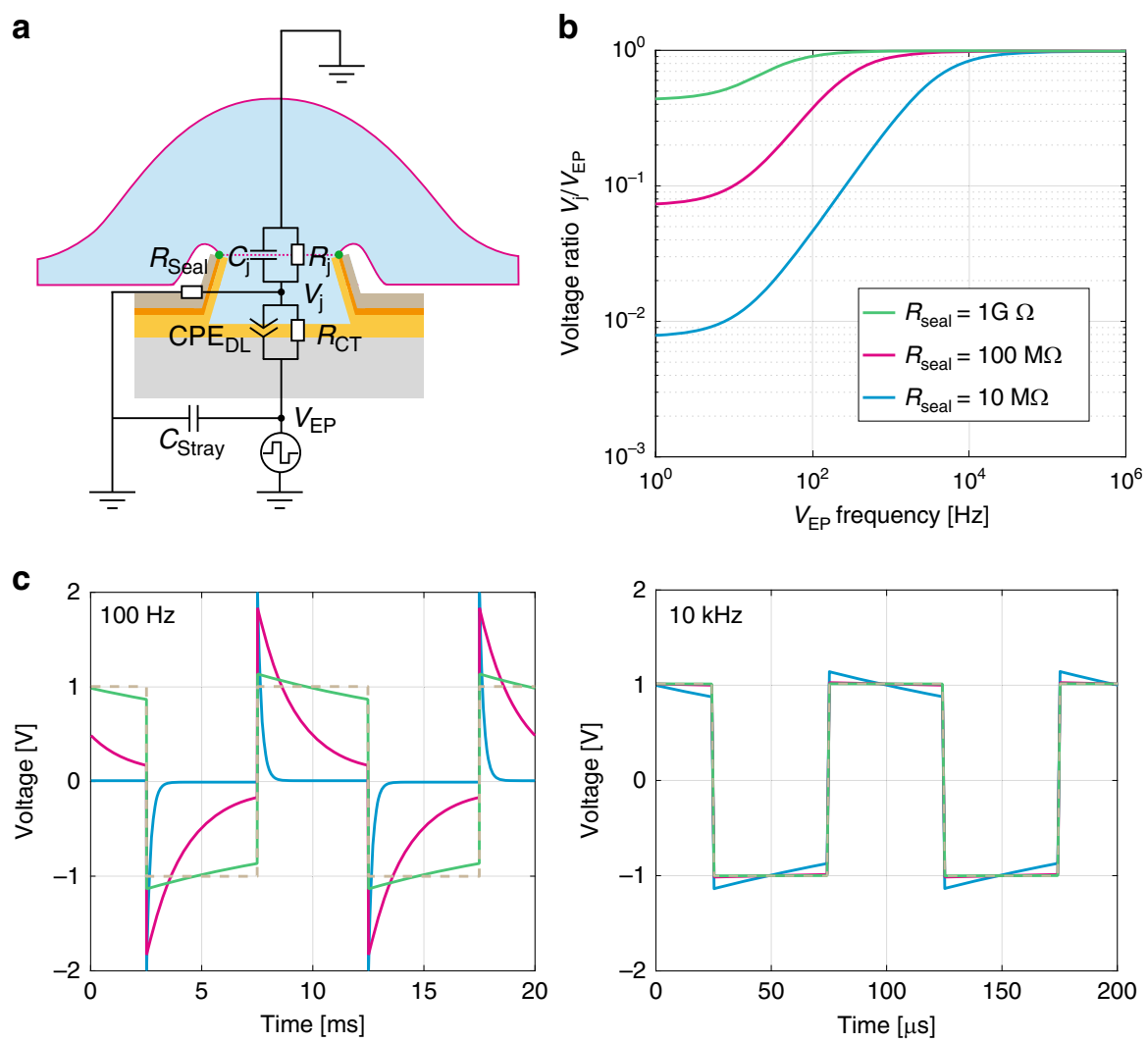

$$
-V_{\mathrm{j}}, R_{\text {seal }}=10 \mathrm{M} \Omega \quad-V_{\mathrm{j}}, R_{\text {seal }}=100 \mathrm{M} \Omega \quad-V_{\mathrm{j}}, R_{\text {seal }}=1 \mathrm{G} \Omega \quad--V_{\mathrm{EP}}
$$

Fig. 1 Electroporation model. a Equivalent electrical circuit of the cell-nanovolcano interface during electroporation. $C P E_{D L}$ is a constant phase element representing the double layer at the electrode-electrolyte interface, $R_{C T}$ is the charge-transfer resistance reflecting the faradaic chargetransfer at the same interface, $C_{j}$ and $R_{j}$ model the junctional capacitance and resistance of the cell membrane, respectively, $R_{\text {seal }}$ is the seal resistance at the cell-electrode interface, and $C_{\text {Stray }}$ represents the capacitive current leaks along the electrical tracks. $\mathbf{b}$ Frequency dependence of the junctional voltage amplitude ratio $\left(V_{j} / V_{E P}\right)$ of an electroporation pulse for different seal resistances $\left(R_{\text {seal }}\right)$. $\mathbf{c} V_{j}$ waveforms in response to square biphasic electroporation pulses ( $V_{\mathrm{EP}}$, stippled line) applied at $100 \mathrm{~Hz}$ (left panel) and $10 \mathrm{kHz}$ (right panel) for different values of $R_{\text {seal }}$ (blue: $10 \mathrm{M} \Omega$; red: $100 \mathrm{M} \Omega$; green: $1 \mathrm{G} \Omega$ )

function of the frequency of the sinusoidal electroporation signal applied at different values of $R_{\text {Seal }}$ ranging from $10 \mathrm{M} \Omega$ to $1 \mathrm{G} \Omega$. At low frequency, the voltage divider determined by $R_{\text {Seal }}$ dominates attenuation because the junctional impedance, especially $R_{j}$, is much higher. Therefore, an electroporation signal oscillating at $1 \mathrm{~Hz}$ is attenuated by a factor of 128 if $R_{\text {Seal }}=10 \mathrm{M} \Omega$ or by a factor of 2 if $R_{\text {Seal }}=1 \mathrm{G} \Omega$. With increasing $V_{\mathrm{EP}}$ frequency, the attenuation factor decreases because the double-layer impedance $\left(Z_{\mathrm{CPE}, \mathrm{DL}}\right)$ and junctional cell membrane impedance $\left(Z_{\mathrm{Cj}}\right)$ decrease. At sufficiently high frequencies, $C_{j}$ replaces $R_{\text {Seal }}$ as the dominant factor, and because $\mathrm{CPE}_{\mathrm{DL}}$ is larger than $C_{j}$, no attenuation is present anymore. Details regarding the analytical computations can be found in the Supplementary Information, Section 2.

Figure 1c shows the shape of $100 \mathrm{~Hz}$ and $10 \mathrm{kHz}$ biphasic square electroporation pulses with amplitude
$V_{\mathrm{EP}}$ together with $V_{j}$ induced across the cell membrane. As opposed to sinusoidal waves, biphasic square waves have the distinct advantage of reaching electroporation-relevant levels of $V_{\mathrm{j}}$ nearly instantaneously and then maintaining these levels during most of the signal period. At $100 \mathrm{~Hz}$ and $R_{\text {Seal }}<1 \mathrm{G} \Omega$, highfrequency components of $V_{\mathrm{EP}}$ are efficiently transmitted to the junctional space, whereas the lowerfrequency parts are drastically attenuated. At $10 \mathrm{kHz}$, the cell membrane voltage follows $V_{\mathrm{EP}}$ with high fidelity even for low seal resistances, thereby ensuring a more efficient electroporation process for a similar overall pulse duration. According to this finding, in this study, we used $10 \mathrm{kHz}$ biphasic electroporation voltage pulses with an overall train duration of $1 \mathrm{~s}$. Higher frequencies were avoided to prevent current leaks through the stray capacitance. 
Influence of the functionalized nanopattern on electrophysiological recordings made with a nanovolcano array

Two features of the volcano-shaped microelectrodes likely determine the quality of the electrophysiological recordings: (a) the high curvature formed by the rim of the nanovolcano is thought to force the cell membrane to follow its shape, thereby increasing the contact area, and (b) the self-assembled alkanethiol monolayer on the gold nanoring exposed on top of the nanovolcano is hypothesized to support tight adhesion of the cell membrane.
Both of these features are expected to increase $R_{\text {Seal }}$ and, hence, raise the signal amplitude. Here, we investigated the relative importance of the two features for establishing a high $R_{\text {Seal }}$. For this purpose, nanovolcanoes with and without thiol-functionalized gold nanorings were produced, as shown schematically in Fig. 2a and by scanning electron micrographs in Fig. 2b. The fabrication process of nanopatterned volcanoes is described in detail in our previous publication $^{24}$. Both types of nanovolcanoes have similar geometries, except for thinner walls in the case of nanovolcanoes that are devoid of a $\mathrm{Ti}-\mathrm{Au}-\mathrm{Ti}$ layer

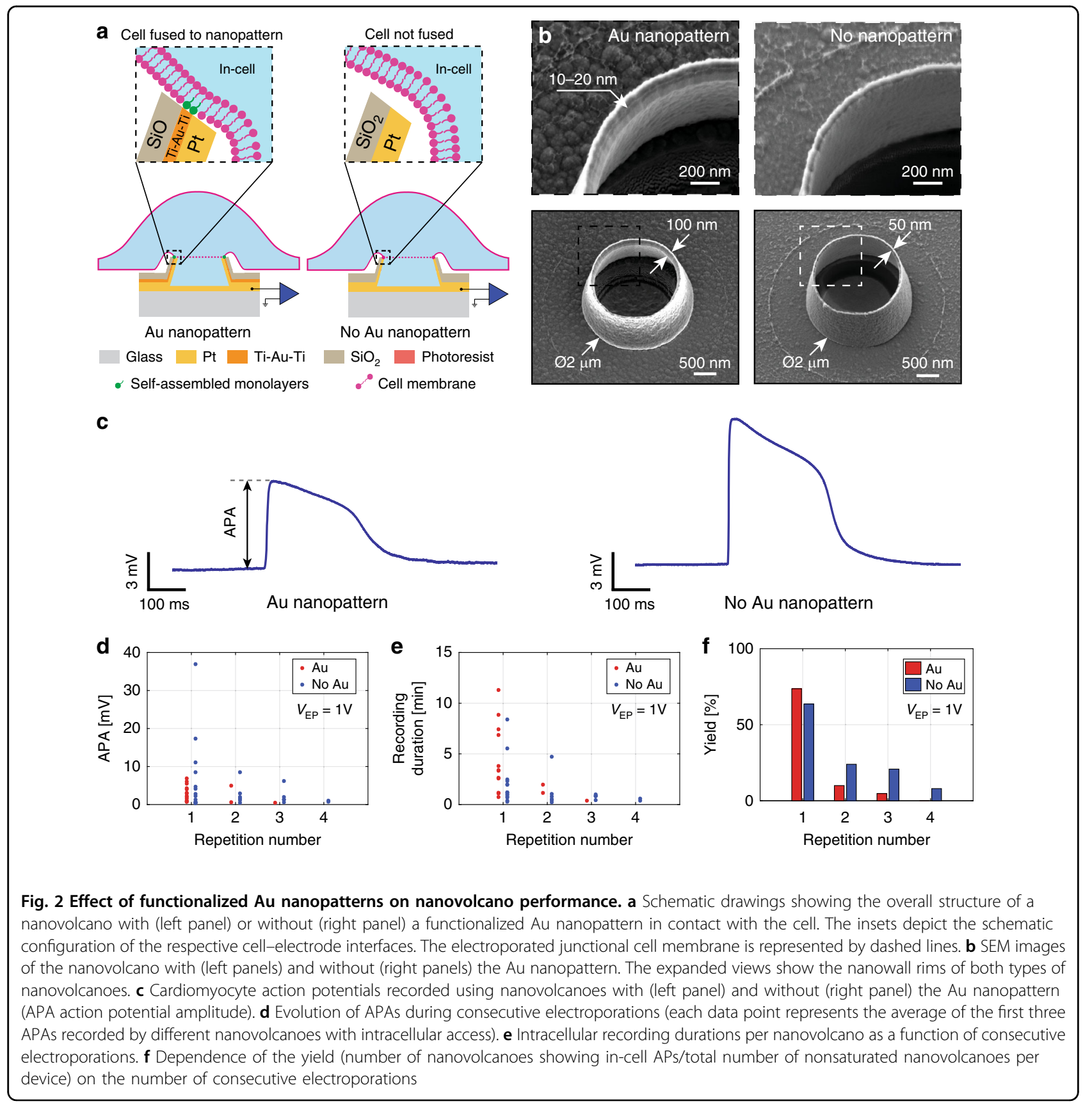


(50 nm instead of $100 \mathrm{~nm}$ ). Each nanovolcano array was composed of 28 recording sites that were laid out according to the schematic drawing shown in the Supplementary Information, Section 1.

The experimental protocol used to compare the performance of the two types of nanovolcanoes consisted of simultaneously applying electroporation pules as defined above ( $1 \mathrm{~V}$ peak-to-peak amplitude, $10 \mathrm{kHz}$ frequency, $1 \mathrm{~s}$ duration) to every microelectrode of devices covered with a monolayer of spontaneously active cardiomyocytes. A phase contrast image of a representative culture of a neonatal rat cardiomyocyte monolayer grown on a nanovolcano array is shown in the Supplementary Information, Section 4. The efficacy of electroporation was assessed by determining the APA, the recording duration, and the yield. A new electroporation was started when all nanovolcanoes of a given device had lost their signals, which resulted in sequential electroporations separated by 5-45 min. Typical examples of successful intracellular recordings of APs obtained by either type of nanovolcano are shown in Fig. 2c. The electrophysiological characteristics presented in the study were extracted from such traces, with the selection criteria defined in the "Methods" section.

An overall analysis of APAs recorded immediately after the given electroporations is presented in Fig. $2 \mathrm{~d}$ for a device with and without functionalized gold rings. Each data point represents the average of APAs of the first three APs recorded by nanovolcanoes with intracellular access. Following the first electroporation (repetition number 1), each type of device reported intracellular activity in 14 channels (yield $\mathrm{Au}_{\mathrm{Au}}: 73.7 \%$, yield $\mathrm{NoAu}_{\mathrm{Nu}}: 63.6 \%$ ) with APAs ranging from 0.8 to $6.8 \mathrm{mV}(3 \pm 2.1 \mathrm{mV}$, $N=14$; nanopatterned) and 0.3 to $36.9 \mathrm{mV}$ (6.6 \pm $10.1 \mathrm{mV}, N=14$; nonpatterned). Signals recorded by all nanovolcanoes with intracellular access declined over time, with a typical example shown in the Supplementary Information, Section 3. After intracellular signals were lost in all channels of a given device, electroporation was repeated (repetition number 2 to 4). APAs as well as the number of channels showing in-cell activity decreased with each further electroporation. After four consecutive electroporation events, the nanopatterned device did not show intracellular activity anymore, whereas in the case of nonpatterned volcanoes, two channels continued to show activity, albeit with very small APAs $(<1 \mathrm{mV})$. Recording durations, i.e., the time until signals completely disappeared, are presented in Fig. 2e. Following the initial electroporation, the recording durations ranged from $44 \mathrm{~s}$ to $11.3 \mathrm{~min}$ ( $4.1 \pm 3.3 \mathrm{~min}, N=14$; patterned devices) and from $16 \mathrm{~s}$ to $8.4 \mathrm{~min}(2.1 \pm 2.2 \mathrm{~min}, N=14$; nonpatterned devices). With each further electroporation, the recording durations decreased. Figure $2 \mathrm{f}$ shows the intracellular access yield as defined by the number of channels showing intracellular activity divided by the total number of nonsaturated channels per device. Similar yields were observed after the first electroporation for both patterned (73.7\%) and nonpatterned (63.6\%) devices. As was the case for APAs and recording durations, the yield decreased with each consecutive electroporation.

The results indicate that nonpatterned nanovolcanoes perform similarly to those with thiol-functionalized gold rings. This finding suggests that the nanovolcano geometry is the dominant parameter in establishing conditions favorable for obtaining intracellular access. For this reason, only nonpatterned devices were used in the remainder of the study.

\section{Effect of the electroporation voltage amplitude on the quality of electrophysiological measurements}

Biphasic square voltage pulses with amplitudes ranging from 1 to $4 \mathrm{~V}$ were applied to nonpatterned nanovolcano arrays covered with spontaneously active cardiomyocyte monolayers to investigate the effect of the electroporation voltage on the yield and quality of the recorded APs. The interval between consecutive electroporations was determined by the time after which none of the microelectrodes of a given device showed intracellular activity anymore (range: $\sim 5$ to $\sim 45 \mathrm{~min}$ ). Figure 3 shows the APAs, recording durations, and yield of successful recordings obtained at increasing electroporation voltages with four different devices. APAs up to $40 \mathrm{mV}$ were successfully recorded, with the highest amplitudes observed at $V_{\mathrm{EP}}=3 \mathrm{~V}$ (cf. Fig. 3a). With each repetition of electroporation, the APAs were further reduced. In the case of $V_{\mathrm{EP}}=4 \mathrm{~V}$, no intracellular activity was detected after the second repetition, suggesting that this voltage caused irreversible cell damage. Similar to the dependence of the APAs on the electroporation voltage, the recording durations depicted in Fig. 3b suggest that intermediate electroporation voltages $(2-3 \mathrm{~V})$ performed best in maximizing periods of intracellular access. With respect to the yield of successful intracellular access following the first electroporation, electroporation voltages ranging from 1 to $3 \mathrm{~V}$ induced access in up to $76.5 \%\left(V_{\mathrm{EP}}=2 \mathrm{~V}\right)$ of the nanovolcanoes (cf. Fig. 3c). Recordings obtained after the first electroporation with $V_{\mathrm{EP}}=2 \mathrm{~V}$ are shown in Fig. $3 \mathrm{~d}$. The yield of gaining intracellular access declined with each additional electroporation with $V_{\mathrm{EP}}=3 \mathrm{~V}$ showing the least decrease. Electroporation voltage amplitudes of $4 \mathrm{~V}$ produced the lowest yields and failed to initiate successful recordings after two electroporation events. Overall, a $V_{\mathrm{EP}}$ of $3 \mathrm{~V}$ performed best in regard to the APAs, recording durations and yield of intracellular access during repeated electroporations.

\section{Long-term on-demand recordings with nanovolcano arrays}

The electroporation protocols were repeated on the same preparations on successive days to investigate the 

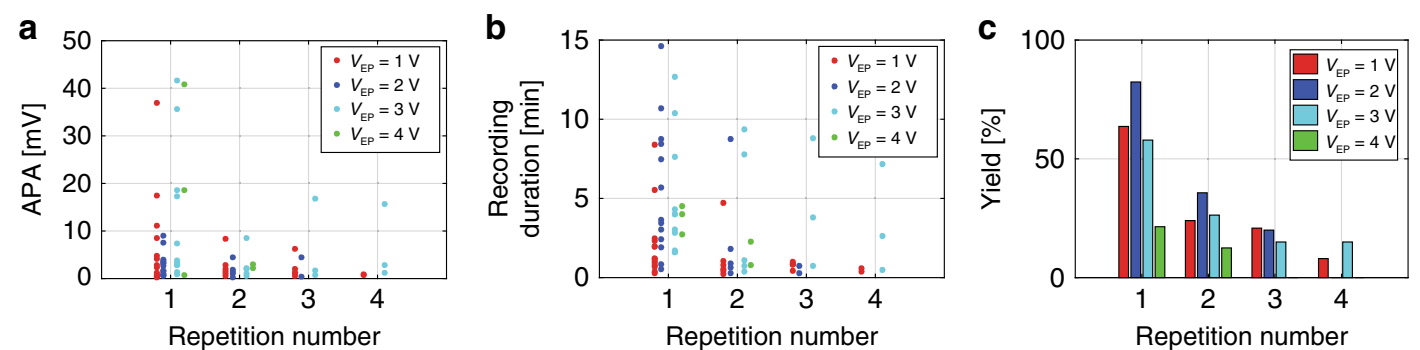

d

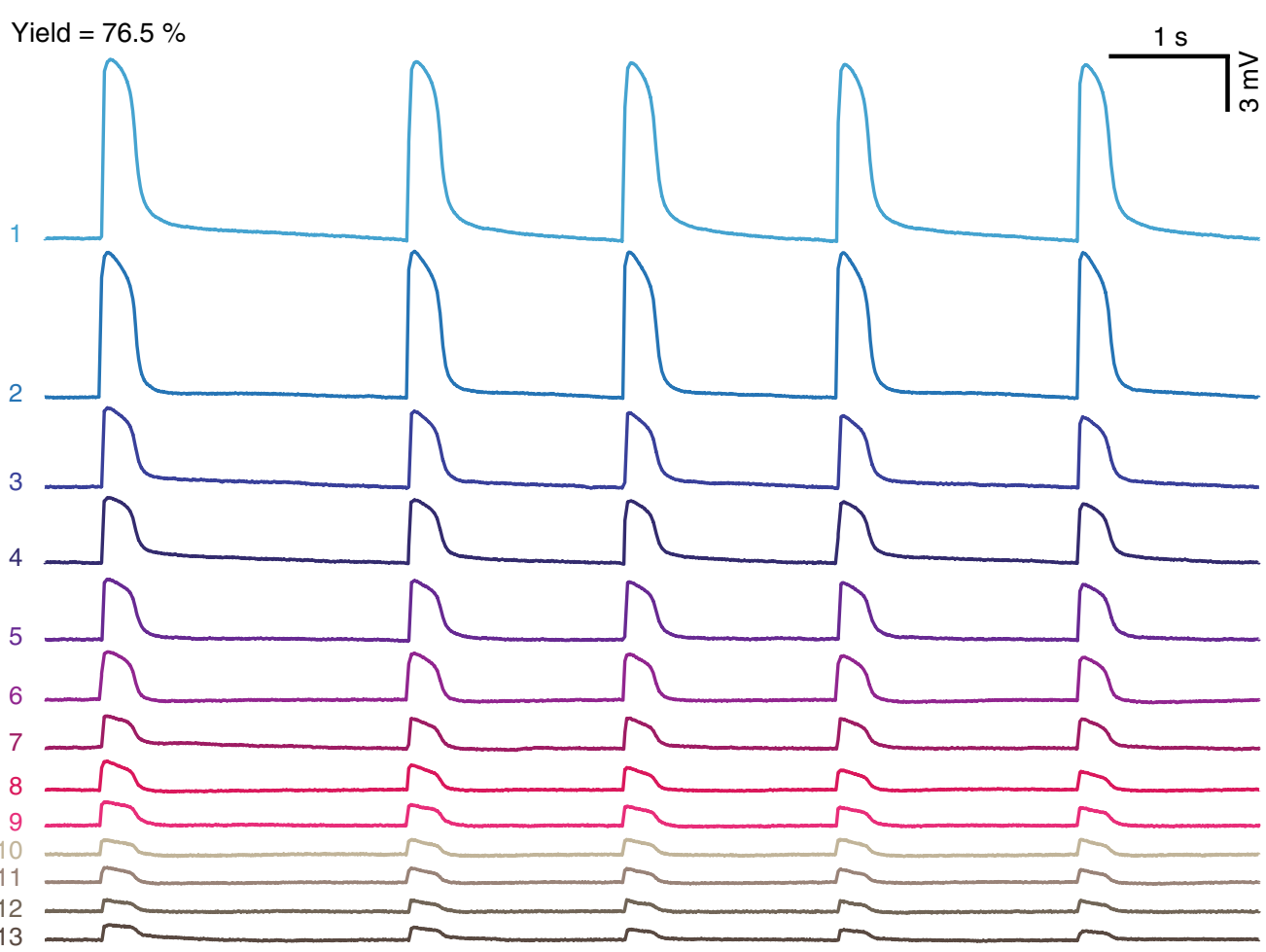

Fig. 3 Effect of electroporation voltage amplitude (VEP) on the success of intracellular nanovolcano recordings. Evolution of the a APA, b recording duration, and $\mathbf{c}$ yield of intracellular access during consecutive electroporations for electroporation voltages ranging from 1 to $4 \mathrm{~V}$. d Simultaneous recording of action potentials from 13 different channels of a nanovolcano array as obtained after the first electroporation with $V_{E P}=2 \mathrm{~V}$ (yield of $\left.76.5 \%\right)$

possibility of using nanovolcano arrays in long-term experiments. The recording characteristics were investigated during four sequential electroporation events $\left(V_{\mathrm{EP}}=3 \mathrm{~V}\right)$ that were repeated after $24 \mathrm{~h}$ (Fig. 4$)$ in one device. Within the two electroporation sequences performed on different days, the APAs, recording durations and yield decreased in a manner qualitatively similar to that of the data shown above for experiments conducted on the same day (Fig. 3). When comparing the electroporation sequence on day 3 to that of day 4, the APAs showed a slight tendency to recover (sequence 4 vs. sequence 5; Fig. 4a), whereas the recording durations further decreased, with the exception of two recordings in sequence 8 (Fig. $4 \mathrm{~b}$ ). The yield showed a decline similar to that observed in other devices during the electroporation sequence on day 3 . On day 4 , the success rate remained rather constant during sequential electroporations; i.e., the average success rate did not differ from that on day 3 (Fig. 4c). Figure 4d shows intracellular APs recorded from a single nanovolcano undergoing multiple electroporations on consecutive days $\left(V_{\mathrm{EP}}=3 \mathrm{~V}\right)$. The corresponding data points are highlighted in magenta in Fig. 4a, b. Figure 4e depicts intracellular recordings of spontaneous APs as recorded on day 4, with a maximal APA $=62 \mathrm{mV}$, an average AP duration at $50 \%$ repolarization $\mathrm{APD}_{50}=166 \pm 0.5 \mathrm{~ms}$ and a maximal upstroke velocity $\mathrm{dV} / \mathrm{dt}_{\max }=22.8 \pm 1.4 \%$ $\mathrm{APA} / \mathrm{ms}\left(V_{\mathrm{EP}}=3 \mathrm{~V}\right.$, repetition 8$)$. 

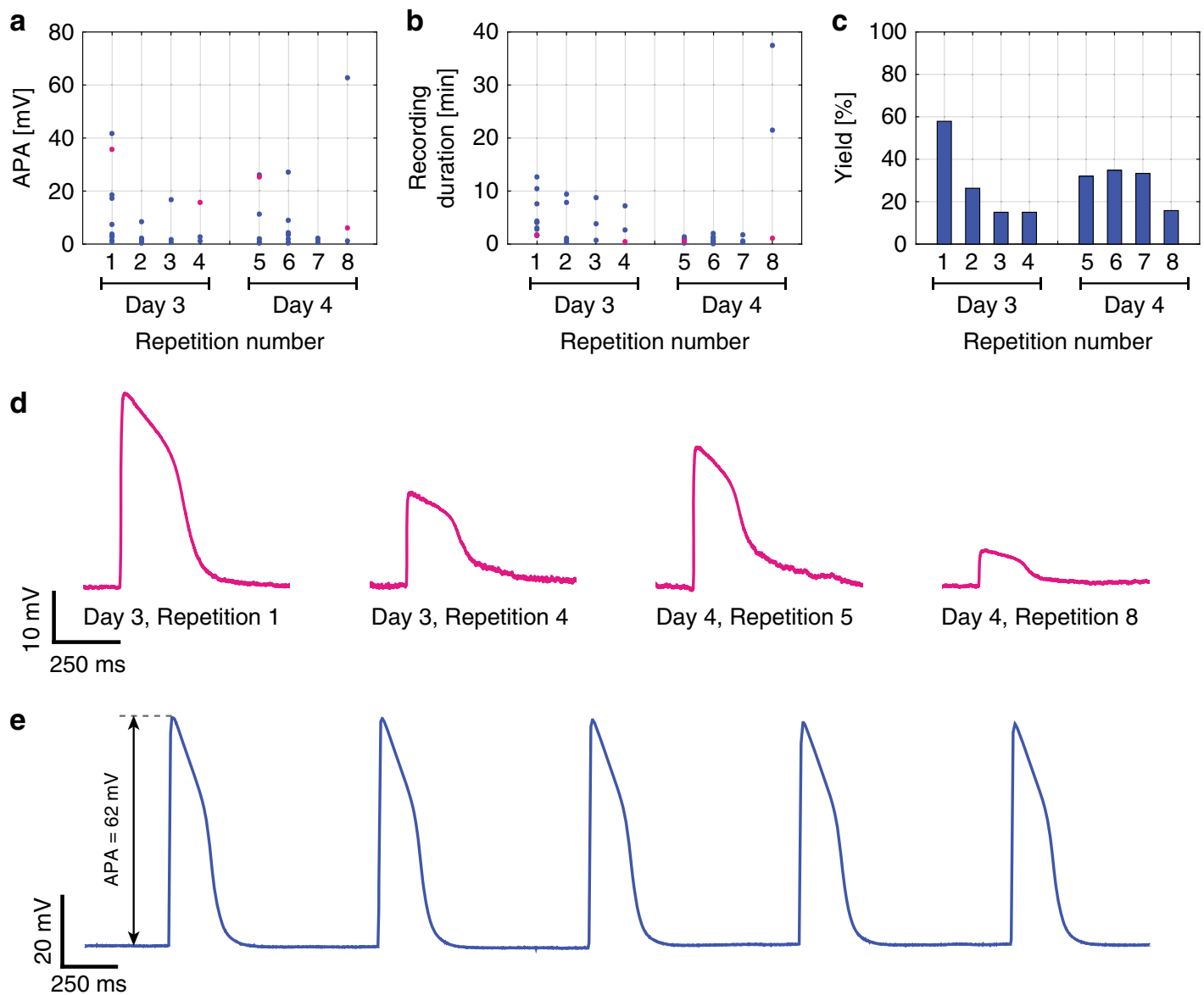

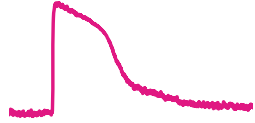

Day 3, Repetition 4

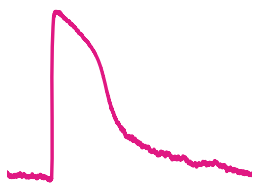

Day 4, Repetition 5

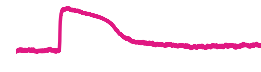

Day 4, Repetition 8

Fig. 4 Long-term recording with nonpatterned nanovolcano arrays. Evolution of the $\mathbf{a}$ APA, $\mathbf{b}$ recording duration, and $\mathbf{c}$ yield with two series of electroporations (four each) performed on successive days $\left(V_{E P}=3 V\right.$ ). $\mathbf{d}$ Action potentials recorded by a single nanovolcano on subsequent days following multiple electroporations. The signals shown correspond to the data points highlighted in magenta in $\mathbf{a}$ and $\mathbf{b}$. e Recording of spontaneous action potentials with an exceptionally high amplitude of $\sim 62 \mathrm{mV}$ on day 4 after the preparation underwent 8 electroporations

Overall, these results demonstrate that nanovolcanoes paired with electroporation permit on-demand intracellular access in cultures of cardiomyocytes for extended periods of time.

\section{Discussion}

We recently introduced nanovolcanoes as a tool to perform multisite intracellular electrophysiological recordings following spontaneous insertion of arrays of nanodevices into networks of excitable cells ${ }^{24}$. Here, we show that controlled electroporation improves the yield of successful intracellular recordings with nanovolcano arrays and permits long-term on-demand measurements of transmembrane electrical activity in excitable tissues.

\section{Optimizing the electroporation parameters}

Optimal electroporation parameters were derived from an analytical model of the cell-electrode interface based on the electrical properties of nanovolcanoes (measured experimentally) and from the general electrical properties of cell membrane characteristics ${ }^{31}$. The model permitted an estimation of the optimal electroporation signal frequency for efficient perforation of the cell membrane at low voltages. The calculation was based on seal resistances reported previously for similar micronanoelectrodes (a few hundred megaohms) ${ }^{17,32}$. This calculation predicted that an electroporation frequency of $10 \mathrm{kHz}$ is optimal to minimize both the electroporation signal attenuation across the electrode-electrolyte interface and the extent of hydrolysis. This prediction was experimentally confirmed, as electroporation voltage amplitudes as low as $600 \mathrm{mV}$ applied at $10 \mathrm{kHz}$ provided successful intracellular access. Furthermore, the use of $10 \mathrm{kHz}$ biphasic square electroporation pulses induced symmetrical and mainly capacitive charge-transfer at the electrode-electrolyte interface ${ }^{33}$, thereby causing charge balance $^{34}$ and preventing the generation of toxic reactive oxygen species ${ }^{35}$. Both of these effects are known 
to minimize cell damage after electroporation ${ }^{30}$. Electroporation-induced pores in the cell membranes are known to be closely related to pulse duration. While short electroporation pulses $(\sim 100 \mathrm{~ns})$ can damage the intracellular compartment without noticeably affecting the cell membrane ${ }^{36}$, longer electroporation pulses induce higher pore densities in the membrane and, therefore, improve the recording performance ${ }^{37,38}$. In this study, an electroporation pulse duration of $100 \mu \mathrm{s}$ was found to provide an adequate balance between irreversible cell damage induced by electrolysis and a sufficiently high density of electroporation-induced pores.

Even though single electroporation pulses were sometimes found to be sufficient to gain intracellular access with nanovolcanoes, a pulse train with a duration of $1 \mathrm{~s}$ was finally adopted to maximize the yield ${ }^{39}$.

Regarding electroporation voltage amplitudes, $V_{\mathrm{EP}}=$ $3 \mathrm{~V}$ provided an adequate balance between sufficiently reducing the junctional resistance and preventing induction of damage to the seal. As reported in the literature ${ }^{26}$, electroporation damages seal resistances, and the poor recording characteristics reported with $V_{\mathrm{EP}}=4 \mathrm{~V}$ are a likely example of this circumstance.

\section{Performance of functionalized vs. nonfunctionalized nanovolcano tips}

The nonconventional ion-beam etching redeposition ${ }^{40}$ based nanofabrication process underlying nanovolcano manufacturing provided sufficient freedom to develop nonpatterned devices, i.e., nanovolcanoes devoid of the intrawall gold layer. Following thiol functionalization, this gold layer was expected to improve the sealing of the cell membrane with the nanovolcano rim. The nonpatterned devices permitted us to investigate the relative importance of thiol functionalization vs. the high curvature of the nanovolcano rim for establishing successful intracellular access. Nonfunctionalized nanopatterned devices were not used on purpose because the $\mathrm{Ti}-\mathrm{Au}-\mathrm{Ti}$ nanoring itself could have affected the results and, hence, biased the study.

The average APAs recorded after the first electroporation amounted to $3.0 \pm 2.1$ and $6.6 \pm 10.1 \mathrm{mV}$ for nonpatterned and patterned nanovolcanoes, respectively. The corresponding yield and recording durations amounted to $73.7 \% / 4.1 \pm 3.3 \mathrm{~min}$ (nanopatterned) and 63.6\%/2.1 \pm $2.2 \mathrm{~min}$ (nonpatterned; $N=14$ for all data). Whereas the relatively large variability of values precludes unequivocal conclusions regarding the relative performance of the two types of nanovolcanoes, the overall trend suggests that the geometry of the nanovolcano tip in contact with the cell likely played a dominant role by inducing a tight seal of the cell membrane around the protruding nanostructure ${ }^{27,28}$.

The relatively large variation in APAs, recording durations, and yield among the nanovolcanoes of a given device may be due to several reasons: (i) fabrication-dependent differences in the exact nanostructure of the top of the nanovolcano may have affected the quality of $R_{\text {Seal }}$, with consequences for all assessed parameters; (ii) the position of individual nanovolcanoes with respect to the monolayer of cardiomyocytes may have resulted in microelectrodes being ideally positioned below a given cell and others being situated at cell-cell borders and, hence, having fewer chances to establish a high-quality seal ${ }^{41}$; and (iii) a few of the nanovolcanoes may have been in contact with noncardiomyocytes (myofibroblasts) that are well known "contaminants" of primary heart cell cultures ${ }^{42}$. These nonexcitable cells are electrotonically coupled to cardiomyocytes and, as a consequence, display passive "APs" with attenuated amplitudes. According to these potential factors inducing variability in recordings, improvements may be made by (i) introducing a polishing procedure to obtain reproducible and seal-promoting flat surfaces of the nanovolcanoes, (ii) controlling the cell positioning with respect to the nanovolcanoes with hydrodynamic traps ${ }^{43,44}$, and (iii) producing cardiomyocyte-only cell cultures by, e.g., fluorescence-activated cell sorting. Moreover, probing signals to investigate the cell-electrode coupling quality might be applied and the results used to compensate APAs according to a calibration curve established beforehand.

Overall, and apart from performing at least similar to, if not better than, patterned nanovolcano devices, nonpatterned nanovolcano devices have the distinct advantage of being based on a simplified preparation procedure that facilitates their production and storage in the case of a potential scale-up.

\section{Effects of repeated electroporations}

Compared with previous results obtained with nanovolcanoes gaining spontaneous intracellular access (maximal APA of $20 \mathrm{mV}$; maximal yield of $30 \%$; maximal recording duration of $66 \mathrm{~min})^{24}$, the recording success was substantially improved by electroporation. APAs up to $40 \mathrm{mV}$ were frequently observed, with one recording showing an APA of $62 \mathrm{mV}$, which corresponds to $2 / 3$ of a nonattenuated cardiomyocyte $\mathrm{APA}^{45}$. In addition, the yield of nanovolcanoes reporting intracellular electrical activity more than doubled to $76.5 \%$. Similar to the decrease in APAs in the case of nanovolcanoes gaining spontaneous access to the cell interior, the APAs declined after electroporation, indicating that, for both modalities, the cell membrane spanning the opening of the nanovolcano tended to reseal. That electroporation only provides intermittent intracellular access has been previously reported $^{23,46}$. With each consecutive electroporation, the APAs, recording duration and yield showed a decline: when comparing the first with the last (fourth) electroporation $\left(V_{\mathrm{EP}}=3 \mathrm{~V}\right)$, the APAs decreased by $\sim 50 \%$, the recording durations by $\sim 30 \%$ and the yield by $\sim 75 \%$. The 
decrease in APAs may be explained by deterioration of the cell-nanovolcano contact and, hence, a decrease in $R_{\text {Seal. }}$ Interestingly, when pausing electroporation for $24 \mathrm{~h}$, both the APAs and yield showed a slight recovery, suggesting partial restitution of the cell-electrode interface.

\section{On-demand intracellular access for long-term recordings}

Compared with our previous work, the possibility of reopening membranes by electroporation increased the apparent overall recording duration by permitting discontinuous on-demand intracellular access over consecutive days. This result suggests that electroporation performed with nanovolcanoes is limited to the small membrane patch defined by the nanovolcano geometry and therefore permits gentle access to the intracellular space without altering cell viability. On average (for $V_{\mathrm{EP}}=3 \mathrm{~V}$, repetition 1), intracellular access lasted $4.9 \pm$ $3.7 \min (N=11 ; 20 \pm 18.2 \min , N=3$, max. $38 \min$ for $V_{\mathrm{EP}}=3 \mathrm{~V}$, repetition 8) before the cell membrane resealed. Throughout this period, the APAs declined with time, therefore limiting the use of nanovolcanoes for applications where long-term APA stability is required. Unstable recordings following electroporation are unfortunately a common issue faced by current micronanoelectrode arrays. A possible explanation for cell membrane resealing proposed by Spira et al. suggests that the local influx of calcium ions into the cells after electroporation induces exocytosis of intracellular vesicles that patch up the perforated cell membrane ${ }^{47}$. A potential solution to overcome this limitation would be to electroporate holes with larger diameters into the cell membrane, as the self-repair mechanisms are ineffective for larger pore dimensions ${ }^{48}$.

Compared with our previous study where static chargeinduced electroporation was suggested as a main penetration mechanism, the recording duration following controlled electroporation is shorter $(4.9 \pm 3.7 \mathrm{~min}, N=$ 11 ; vs. $18.2 \pm 21 \mathrm{~min}, N=16$ ). This result may be explained by assuming that static charge-induced electroporation leads to larger pores, which take more time to reseal. Potentially, nanovolcanoes with higher walls may increase the seal resistance and therefore minimize the calcium influx triggering self-repair mechanisms. Alternatively, longer nonattenuated electroporation pulses across the cell membrane may induce larger holes and improve the recording duration time without triggering adverse electrolysis reactions.

Despite the problems associated with APA attenuation and membrane resealing, repeated measurements on the same site of given preparations are essential advantage over conventional patch-clamp measurements of APs, as it has the potential for screening pharmacological compounds at high throughput and over extended periods of time for cases where AP shapes rather than absolute values of transmembrane voltage changes are of interest. For example, nanovolcanoes permit screening of druginduced changes in AP duration ${ }^{24}$, which is essential for drug development because prolongation or shortening of APDs is a well-established factor contributing to the precipitation of cardiac arrhythmias. Accordingly, new drug candidates are extensively tested for this unwanted side effect. Because nanovolcano arrays enable quantitative measurements of AP durations, their application in drug development seems accordingly feasible.

\section{Comparison to other micro-nanotechnology-based electrode arrays granting intracellular access}

Micro-nanotechnology-based electrode arrays aim to assess the electrophysiological characteristics of networks of excitable cells over extended periods of time with recording qualities approaching those of standard intracellular measurements. Table 1 compares the respective performance of recently presented micro-nanotechnologybased MEAs. In all experiments, data were collected from primary rat cardiomyocyte cell cultures with intracellular access induced by electro- or opto-poration. By comparison, nanotube-like electrodes composed of IrOx enabled the longest continuous intracellular recordings ( 100 min), followed by nanovolcanoes, plasmonic metaelectrodes, nanoneedle,s and micromushrooms. The APAs were largest in the nanovolcanoes, while the maximal yield was best in plasmonic metaelectrodes, followed closely by nanovolcanoes. By contrast to the other technologies, APs measured with nanovolcano arrays did not suffer from

Table 1 Summary of features of transmembrane voltage measurements with micro-nanoelectrodes in primary rat cardiomyocyte monolayers

\begin{tabular}{|c|c|c|c|c|c|c|}
\hline \multirow[t]{2}{*}{ Insertion method } & \multirow[t]{2}{*}{ Technology } & \multirow[t]{2}{*}{ Max. APA $[\mathrm{mV}]$} & \multicolumn{2}{|l|}{ Max. duration } & \multirow[t]{2}{*}{ Max. yield [\%] } & \multirow[t]{2}{*}{ Distortion/bandwidth $[\mathrm{Hz}]$} \\
\hline & & & Single access [min] & Multiple access & & \\
\hline \multirow[t]{4}{*}{ Electroporation } & Nanovolcano & 62 & $66^{24}$ & Days & 76.5 & No/0-8000 \\
\hline & Nanoneedle ${ }^{17}$ & 20 & 20 & Days & $\sim 30$ & Yes/1-5000 \\
\hline & Micromushroom ${ }^{19}$ & 6 & 10 & Days & - & Yes/1-10,000 \\
\hline & IrOx nanotube $\mathrm{e}^{23}$ & 15 & $\sim 100$ & Days & 10 & Yes/0.01-3000 \\
\hline Optoporation & Plasmonic metaelectrode ${ }^{50}$ & 5 & 40 & Minutes & 85.4 & - \\
\hline
\end{tabular}


high-pass filtering distortion due to the recording system and therefore can be exploited, e.g., for pharmacological studies aiming at detecting side effects of drugs on the AP shape in general and AP duration in particular.

\section{Limitations of the study and outlook}

In this study, the electrophysiological recording performance of two different nanovolcano architectures was investigated under different electroporation modalities, with one device being used per condition. This low number does not allow for a systematic statistical comparison of the data. The unequivocal results obtained, however, permit us to draw qualitative conclusions and detect trends when comparing the micro-nanoelectrode devices.

Future developments of nanovolcano arrays may focus on a systematic optimization of the nanovolcano geometry to further optimize its performance. As demonstrated in this work, the protruding nanovolcano geometry is the decisive factor in forming a tight seal at the cell-electrode interface. It is therefore essential to optimize its shape, dimensions, and material composition in a systematic manner to achieve improved recording performance, especially in terms of recording duration. In the literature, hollow nanoelectrodes composed of IrOx showed intracellular recording durations up to $100 \mathrm{~min}$, much longer than those of $\mathrm{Au}$ pillars with similar dimensions ${ }^{23}$. As a further improvement, nanovolcanoes may be directly manufactured on integrated CMOS amplifiers in order to maximize signal-to-noise ratios and facilitate the electroporation procedure. This approach would also permit an increase in the density of nanovolcanoes per device, which is now limited by the electrical tracks. Such an approach has been previously described for nanoelectrodes (1024 recording sites with dedicated amplifiers) ${ }^{17}$. Apart from producing larger datasets at minimized experimental cost, a highresolution array would extend the system capabilities in the context of permitting spatially resolved measurements of electrical activation patterns.

\section{Conclusion}

In this work, we demonstrate that electroporation significantly improves the recording characteristics of transmembrane APs measured with nanovolcano arrays. An analytical model is proposed to predict the electroporation voltage attenuation across the electrode-electrolyte interface and suggests optimal electroporation parameters to perforate cell membranes with low-voltage amplitudes. Electroporation is shown to drastically improve the quality of the electrophysiological recordings reported with nanovolcano arrays in terms of the APAs, recording duration, and yield. The signal quality is shown to be independent of the presence of functionalized nanopatterns incorporated into the tip of the nanovolcano. This finding indicates that the "sharp" geometry of the top of the nanovolcanoes is sufficient to establish optimal seal conditions at the cell-electrode interface. Importantly, electroporation enables on-demand intracellular access during consecutive days. This result suggests that nanovolcanoes have no detrimental effects on cells that they are in contact with, and it opens the possibility to follow electrical activity over periods of time that are relevant for assessing developmental aspects of the preparations under investigation.

\section{Methods \\ Microfabrication}

Nanovolcano arrays were manufactured following the nanofabrication process described in detail in our previous work $^{24}$. Briefly, a standard fused silica wafer $(100 \mathrm{~mm}$ in diameter, $550-\mu \mathrm{m}$ thick) was covered with a multilayered metal stack by successive evaporations prior to being coated by a 150 -nm-thick layer of sputtered $\mathrm{SiO}_{2}$. For nanovolcanoes on which a thiol pattern was self-assembled ("patterned nanovolcanoes"), the multilayered stack consisted of $\mathrm{Ti}-\mathrm{Pt}-\mathrm{Ti}-\mathrm{Au}-\mathrm{Ti}-\mathrm{SiO}_{2}(10-250-50-50-50-150 \mathrm{~nm})$. For nanovolcano arrays without the self-assembled thiol pattern ("nonpatterned nanovolcanoes"), a stack of $\mathrm{Ti}-\mathrm{Pt}-\mathrm{SiO}_{2}$ (10-250-150 nm) was used instead. Openings $2 \mu \mathrm{m}$ in diameter were patterned into a 2- $\mu$ m-thick AZ nLOf 2020 negative photoresist layer (MicroChemicals, Germany) by direct laser writing. $\mathrm{Ar}^{+}$ion-beam etching redeposition was used to manufacture the nanovolcano sidewalls ${ }^{40}$. Next, $3-\mu \mathrm{m}$-wide electrical tracks that connected every nanovolcano to its interfacing pad were patterned by photolithography and conventional ion-beam etching. Finally, a 4.5- $\mu \mathrm{m}$-thick SU8 insulating layer was coated and patterned by direct laser writing, providing $20-\mu \mathrm{m}$-diameter openings surrounding each microelectrode of the wafer.

\section{Device preparation}

Nanovolcano arrays were assembled with a glass ring forming the cell culture well using polydimethylsiloxane (Sylgard 184 Silicone Elastomer kit 10:1; Dow Corning, USA) as glue and cured for $2 \mathrm{~h}$ at $80^{\circ} \mathrm{C}$. Nanopatterned arrays were sterilized for $30 \mathrm{~s}$ by $\mathrm{O}_{2}$ plasma $(100 \mathrm{~W}, 650$ mTorr; Diener Electronic, Germany) prior to being submerged for $1 \mathrm{~h}$ in a $20 \mathrm{mmol} / \mathrm{L}$ hexanethiol solution in pure ethanol for self-assembled monolayer formation. After ethanol rinsing, the microelectrode arrays were thoroughly rinsed with sterile deionized water. As previously demonstrated, hexanethiols do not attach to the Pt electrode ${ }^{26}$. For nonpatterned devices, the hexanethiol solution was replaced by pure ethanol. Finally, the substrate was coated with collagen type IV (C5533, Sigma, Germany) before cell seeding. Only new devices were used during this study. 


\section{Isolation and culture of primary rat cardiomyocytes}

Primary neonatal rat (Wistar, 1-day old) ventricular cardiomyocytes were isolated and cultured following procedures previously described in detail ${ }^{49}$. Experiments were carried out in compliance with federal guidelines for animal experimentation under license BE27/17 of the Bernese Veterinary Department. In brief, hearts of 4-10 neonatal rats were excised, and ventricles were separated from atria in cold Hank's balanced saline solution without $\mathrm{Ca}^{2+}$ and $\mathrm{Mg}^{2+}$ (3-02F29-I, BioConcept, Switzerland) supplemented with trypsin (0.1\%; Sigma, T4674) and pancreatin $(120 \mu \mathrm{g} / \mathrm{mL}$; Sigma, P3292). Ventricles were minced using scissors and subjected to 4 or 5 consecutive dissociation cycles in an agitated container maintained at $36^{\circ} \mathrm{C}$. After each cycle, the supernatant was removed and stored on ice. New dissociation solution was added to the remaining tissue pieces, and the next dissociation cycle was started. The dissociated cells were spun down and resuspended in M199 medium (M7653, Sigma) supplemented with penicillin (20 U/mL; Sigma, P7794), vitamin $\mathrm{B}_{12}(2 \mu \mathrm{g} / \mathrm{mL}$; Sigma, V2876), vitamin C $(18 \mu \mathrm{mol} / \mathrm{L}$; Sigma A-4544), epinephrine (10 $\mu \mathrm{M}$; Sigma, E4250), bromodeoxyuridin (100 $\mu \mathrm{mol} / \mathrm{L}$; Sigma, B-5002), L-glutamine (680 $\mu \mathrm{M}$; Sigma, G7513), and neonatal calf serum (S 0125, Biochrom, Bioswisstec, Switzerland). Cardiomyocytes were purified by differential preplating in cell-culture flasks. After $2 \mathrm{~h}$, the supernatant containing mostly cardiomyocytes was removed from the flasks, and the cell number was determined using a hemocytometer. Dilution was adjusted to achieve a seeding density of $\sim 3500$ cardiomyocytes per $\mathrm{mm}^{2}$. Preparations were incubated for $24 \mathrm{~h}$ at $36^{\circ} \mathrm{C}$ in a humidified atmosphere containing $0.8 \% \mathrm{CO}_{2}$. After $24 \mathrm{~h}$, the cell-culture medium was replaced, and the serum concentration was lowered from 10 to 5\%. Thereafter, the cell-culture medium was replaced every other day.

\section{Electroporation procedure}

Prior to the first electroporation, a control recording was performed to guarantee the absence of intracellular activity due to static charge-induced electroporation ${ }^{24}$. Thereafter, before initiating the electrophysiological recordings, biphasic voltage pulses with a period of $100 \mu \mathrm{s}$ and an amplitude ranging from 1 to $4 \mathrm{~V}$ with respect to the four reference electrodes integrated in the device were simultaneously applied to every channel of the device using an A-M Systems Model 2100 pulse stimulator (A-M Systems, USA) for an overall duration of $1 \mathrm{~s}$.

\section{Electrophysiology}

Electrophysiological experiments were started $48 \mathrm{~h}$ after cell seeding. At this time, the cardiomyocytes had formed a uniform cell monolayer that exhibited spontaneous synchronized electrical activity, and contamination by proliferating noncardiomyocytes was still moderate.
Experiments were performed in a dry incubator $\left(37^{\circ} \mathrm{C} ; 0.8 \%\right.$ $\left.\mathrm{CO}_{2}\right)$ using a DC-coupled HS-36 headstage $\left(R_{\text {in }}=1 \mathrm{~T} \Omega\right.$, $C_{\text {in }}=2 \mathrm{pF}$; bandwidth $\left.=0-8 \mathrm{kHz}\right)$ together with a Digital Lynx SX acquisition system (Neuralynx, USA). Preparations were covered with a lid to prevent evaporation.

\section{Data analysis}

A custom-made MATLAB2016a (MathWorks, USA) script was developed to extract the electrophysiological parameters of interest from the recorded signals (APA, $A P D_{50}$, and $\left.d V / d t_{\text {max }}\right)$ as well as the recording duration per channel and intracellular yield per device. Every electrical trace analyzed and presented in this work corresponds to the nonfiltered raw data.

To objectively define the duration of intracellular recording per channel, an AP was considered intracellular until its amplitude decreased below $250 \mu \mathrm{V}$ or until the maximum of the first derivative thereof became smaller than three times the absolute value of its minimum, which is typical for extracellular recordings.

Recordings from channels showing saturation were excluded from the analysis. Saturation occurring in a few channels was likely the result of the combination of the relatively large impedance of the nanovolcanoes with the DC-coupled headstage of the recording system.

Values are given as the mean \pm standard deviation (mean $\pm \mathrm{SD}$ ) throughout the manuscript.

\section{Acknowledgements}

This work was partially funded by the Swiss National Science Foundation (grant number 200021_175943 to P.R. and 310030_169234 to S.R.). The authors thank the EPFL Center of Micronanotechnology (CMi), Atelier Pour le Routage et la Fabrication de Circuit Imprimés (ACI), and Atelier de Fabrication Additive (AFA) staff for their outstanding support and availability. Special thanks to Christian Dellenbach for his help with the electrical interfacing and Regula Flückiger Labrada for her excellent cell-culture work (both from Bern University).

\section{Author contributions}

P.R., S.R., and B.X.E.D. conceived the project, B.X.E.D. and N.M. manufactured the nanovolcano arrays in a clean room, E.d.C. handled the cardiomyocyte isolation and culture, B.X.E.D. and E.d.C. performed the electrophysiological experiments, B.X.E.D., E.d.C., and S.R. interpreted the results, P.R. and A.B. supervised the technological part of the project and S.R. supervised the biological part. All authors discussed the results and contributed to the final manuscript.

\section{Conflict of interest}

The authors declare that they have no conflict of interest.

Supplementary information accompanies this paper at https://doi.org/ 10.1038/s41378-020-0178-7.

Received: 28 February 2020 Revised: 11 April 2020 Accepted: 5 May 2020 Published online: 24 August 2020

\footnotetext{
References

1. Stämpfli, R. Reversible electrical breakdown of the excitable membrane of a Ranvier node. An. da Academia Brasileira de. Ciências 30, 57-63 (1958).

2. Sale, A. J. H. \& Hamilton, W. A. Effects of high electric fields on microorganisms. I. Killing of bacteria and yeasts. BBA Gen. Subj. 148, 781-788 (1967).
} 
3. Kotnik, T. et al. Electroporation-based applications in biotechnology. Trends Biotechnol. 33, 480-488 (2015).

4. Markov, M. S., Pakhomov, A. G. \& Miklavcic, D. Advanced electroporation techniques in biology and medicine, (CRC Press Book, 2010).

5. Neumann, E., Schaefer-Ridder, M., Wang, Y. \& Hofschneider, P. H. Gene transfer into mouse lyoma cells by electroporation in high electric fields. EMBO J. 1, 841-845 (1982)

6. Wong, T. K. \& Neumann, E. Electric field mediated gene transfer. Biochem. Biophys. Res. Commun. 107, 584-587 (1982).

7. André, F. \& Mir, L. M. DNA electrotransfer: Its principles and an updated review of its therapeutic applications. Gene Ther. 11, 33-42 (2004).

8. Zhan, Y., Sun, C., Cao, Z., Bao, N., Xing, J. \& Lu, C. Release of intracellular proteins by electroporation with preserved cell viability. Anal. Chem. 84, 8102-8105 (2012).

9. Ching, C. T. S., Fu, L. S., Sun, T. P., Hsu, T. H. \& Chang, K. M. Use of electroporation and reverse iontophoresis for extraction of transdermal multibiomarkers. Int. J. Nanomed. 7, 885-894 (2012).

10. Sack, M. et al. Research on industrial-scale electroporation devices fostering the extraction of substances from biological tissue. Food Eng. Rev. 2, 147-156 (2010).

11. Mir, L. M. \& Orlowski, S. Mechanisms of electrochemotherapy. Adv. Drug Deliv. Rev. 35, 107-118 (1999).

12. Jaroszeski, M. J., Gilbert, R. \& Heller, R. Electrochemotherapy: an emerging drug delivery method for the treatment of cancer. Adv. Drug Deliv. Rev. 26, 185-197 (1997).

13. Heller, R., Gilbert, R. \& Jaroszeski, M. J. Clinical applications of electrochemotherapy. Adv. Drug Deliv. Rev. 35, 119-129 (1999).

14. Yajuan, X., Xin, L. \& Zhiyuan, L. A comparison of the performance and application differences between manual and automated patch-clamp techniques. Curr. Chem. Genom. 6, 87-92 (2013).

15. Robinson, J. T. et al. Vertical nanowire electrode arrays as a scalable platform for intracellular interfacing to neuronal circuits. Nat. Nanotechnol. 7, 180-184 (2012).

16. Robinson, J. T., Jorgolli, M. \& Park, H. Nanowire electrodes for high-density stimulation and measurement of neural circuits. Front. Neural Circuits 7, 38 (2013).

17. Abbott, J. et al. CMOS nanoelectrode array for all-electrical intracellular electrophysiological imaging. Nat. Nanotechnol. 12, 460-466 (2017).

18. Abbott, J. et al. A nanoelectrode array for obtaining intracellular recordings from thousands of connected neurons. Nat. Biomed. Eng. 4, 232-241 (2020).

19. Fendyur, A. \& Spira, M. E. Toward on-chip, in-cell recordings from cultured cardiomyocytes by arrays of gold mushroom-shaped microelectrodes. Front. Neuroeng. 5, 1-10 (2012).

20. Hai, A. \& Spira, M. E. On-chip electroporation, membrane repair dynamics and transient in-cell recordings by arrays of gold mushroom-shaped microelectrodes. Lab Chip 12, 2865-2873 (2012).

21. Shmoel, N. et al. Multisite electrophysiological recordings by self-assembled loose-patch-like junctions between cultured hippocampal neurons and mushroom-shaped microelectrodes. Sci. Rep. 6, 27110 (2016).

22. Spira, M. E., Shmoel, N., Huang, S. H. M. \& Erez, H. Multisite attenuated intracellular recordings by extracellular multielectrode arrays, a perspective. Front. Neurosci. 12, 212 (2018).

23. Lin, Z. C., Xie, C., Osakada, Y., Cui, Y. \& Cui, B. Iridium oxide nanotube electrodes for sensitive and prolonged intracellular measurement of action potentials. Nat. Commun. 5, 3206 (2014).

24. Desbiolles, B. X. E., De Coulon, E., Bertsch, A., Rohr, S. \& Renaud, P. Intracellular recording of cardiomyocyte action potentials with nanopatterned volcanoshaped microelectrode arrays. Nano Lett. 19, 6173-6181 (2019).
25. Almquist, B. D. \& Melosh, N. A. Fusion of biomimetic stealth probes into lipid bilayer cores. Proc. Natl. Acad. Sci. 107, 5815-5820 (2010).

26. VanDersarl, J. J. \& Renaud, P. Biomimetic surface patterning for long-term transmembrane access. Sci. Rep. 6, 32485 (2016)

27. Dipalo, $M$. et al. Cells adhering to $3 \mathrm{D}$ vertical nanostructures: cell membrane reshaping without stable internalization. Nano Lett. 18, 6100-6105 (2018).

28. Hanson, L., Lin, Z. C., Xie, C., Cui, Y. \& Cui, B. Characterization of the cellnanopillar interface by transmission electron microscopy. Nano Lett. 12, 5815-5820 (2012).

29. Miragoli, M., Salvarani, N. \& Rohr, S. Myofibroblasts induce ectopic activity in cardiac tissue. Circ. Res. 101, 755-758 (2007).

30. Tovar, O. \& Tung, L. Electroporation and recovery of cardiac cell membrane with rectangular voltage pulses. Am. J. Physiol. Heart Circ. Physiol. 263, 1128-1136 (1992).

31. Milo, R. \& Phillips, R. Cell biology by the numbers, (CRC Press Book, 2015).

32. Liu, R. et al. High density individually addressable nanowire arrays record intracellular activity from primary rodent and human stem cell derived neurons. Nano Lett. 17, 2757-2764 (2017).

33. Franks, W., Schenker, I., Schmutz, P. \& Hierlemann, A. Impedance characterization and modeling of electrodes for biomedical applications. IEEE Trans. Biomed. Eng. 52, 1295-1302 (2005).

34. Cogan, S. F. Neural stimulation and recording electrodes. Annu. Rev. Biomed. Eng. 10, 275-309 (2008).

35. Zhang, Y. Cell toxicity mechanism and biomarker. Clin. Transl. Med. 7, 34 (2018).

36. Fox, M. B. et al. Electroporation of cells in microfluidic devices: a review. Anal. Bioanal. Chem. 385, 474-485 (2006).

37. Valero, A. Single cell electroporation on chip, (Wohrmann Print Service, 2006).

38. Gabriel, B. \& Teissié, J. Time courses of mammalian cell electropermeabilization observed by millisecond imaging of membrane property changes during the pulse. Biophys. J. 76, 2158-2165 (1999).

39. Reberšek, M. Beyond electroporation pulse parameters: from application to evaluation. in Handbook of electroporation, Vol. 2, 977-997 (Springer, 2017).

40. Desbiolles, B. X. E., Bertsch, A. \& Renaud, P. lon beam etching redeposition for 3D multimaterial nanostructure manufacturing. Microsyst. Nanoeng. 5, 11 (2019).

41. Santoro, F. et al. Interfacing electrogenic cells with 3D nanoelectrodes: position, shape, and size matter. ACS Nano 8, 6713-6723 (2014).

42. Miragoli, M., Gaudesius, G. \& Rohr, S. Electrotonic modulation of cardiac impulse conduction by myofibroblasts. Circulation Res. 98, 801-810 (2006).

43. Di Carlo, D., Wu, L. Y. \& Lee, L. P. Dynamic single cell culture array. Lab Chip 6, 1445-1449 (2006).

44. Yesilkoy, F. et al. Highly efficient and gentle trapping of single cells in large microfluidic arrays for time-lapse experiments. Biomicrofluidics 10, 014120 (2016).

45. Rohr, S., Schölly, M. \& Kléber, A. G. Patterned growth of neonatal rat heart cells in culture. Circ. Res. 68, 114-130 (1991).

46. Xie, C., Lin, Z., Hanson, L., Cui, Y. \& Cui, B. Intracellular recording of action potentials by nanopillar electroporation. Nat. Nanotechnol. 7, 185-190 (2012).

47. Chiappalone, M., Pasquale, V. \& Monica, F. In vitro neuronal networks, (Springer, 2019).

48. Joshi, R. P. \& Schoenbach, K. H. Mechanism for membrane electroporation irreversibility under high-intensity, ultrashort electrical pulse conditions. Phys. Rev. E 66, 4 (2002).

49. Rohr, S., Flückiger-Labrada, R. \& Kucera, J. P. Photolithographically defined deposition of attachment factors as a versatile method for patterning the growth of different cell types in culture. Pflug. Arch. Eur. J. Physiol. 446, 125-132 (2003).

50. Dipalo, M. et al. Plasmonic meta-electrodes allow intracellular recordings at network level on high-density CMOS-multi-electrode arrays. Nat. Nanotechnol. 13, 965-971 (2018). 